Meta

Journal des tradlucteurs

Translators' Journal

\title{
Le traducteur et l'assurance sur la vie
}

\section{Jean-Paul de Grandpré}

Volume 11, numéro 2, juin 1966

URI : https://id.erudit.org/iderudit/010471ar

DOI : https://doi.org/10.7202/010471ar

Aller au sommaire du numéro

Éditeur(s)

Les Presses de l'Université de Montréal

ISSN

0026-0452 (imprimé)

1492-1421 (numérique)

Découvrir la revue

Citer cet article

de Grandpré, J.-P. (1966). Le traducteur et l'assurance sur la vie. Meta, 11(2),

70-74. https://doi.org/10.7202/010471ar d'utilisation que vous pouvez consulter en ligne.

https://apropos.erudit.org/fr/usagers/politique-dutilisation/ 


\section{le traducteur \\ et l'assurance sur la vie}

Il y avait en 1964 plus de huit millions de polices d'assurance sur la vie en cours au Canada, et la masse des assurances correspondantes se montait à près de soixante-trois milliards de dollars. C'est là un phénomène social et économique de la plus haute importance. Qui de nos jours ne s'en remet pour sả sécurité à «sa » police et à la valeur de rachat qu'elle garantit ? Qui n'ambitionne, s'il ne l'a déjà fait, d'ajouter à son contrat un avenant d'assurance en cas de décès par accident? de s'assurer d'une indemnité pour le jour où il pourrait être frappé d'invalidité ?

Et pourtant, l'éveil de la conscience linguistique que l'on constate un peu partout au pays s'est à peine manifesté jusqu'à maintenant sur le plan des assurances sur la vie. Il suffit, pour s'en convaincre, de lire le texte français des contrats et autres imprimés publiés par nos organismes d'assurance sur la vie: trop souvent, la phrase n'est française ni dans sa structure ni dans le vocabulaire utilisé ; les incorrections et les impropriétés de termes y abondent; ils sont truffés de calques injustifiés et, par voie de conséquence, d'anglicismes et de barbarismes.

Faut-il, par exemple, traduire occupational ratings ? On dit sans sourciller «surprimes occupationnelles» ou, encore plus péniblement, « cotes attri- 
buables à l'occupation », au lieu de surprimes professionnelles. On rend actuarial assistant par « assistant actuariel », plutôt que par adjoint d'actuariat. On bute sur juvenile insurance: on oublie que assurance d'enfant ferait l'affaire et l'on écrit soit «assurance juvénile», sans se soucier du sens affectif qui ne convient guère pour désigner une catégorie $d$ 'assurance, soit «assurance infantile »... N'avons-nous pas vu dans l'un des contrats d'une grande compagnie le mot «bénéfice » désigner en même temps la quote-part de bénéfices et les sommes garanties: un seul mot pour nommer deux choses différentes, et dans un même texte. Voilà qui est fort !

En raison même de ces erreurs de français, ces imprimés n'ont souvent de sens que pour les initiés: les interpréter n'est pas chose facile. Si l'on y parvient, c'est souvent à cause d'une généralisation de l'erreur et à cause d'une certaine connaissance de l'anglais, qui nous est commune à tous. Plusieurs employés avouent que, pour eux, écrire une lettre en français est un supplice. D'autres soucis devraient également les habiter, et ne pas être étrangers non plus aux traducteurs (et aux trésoriers !), car les dangers que présente cet état de choses sont réels.

Les polices d'assurance sur la vie font appel à des notions complexes d'ordre actuariel et juridique. Les sommes en jeu sont souvent considérables, et portent sur des délais indéterminés: il est possible que le risque représenté par une police souscrite maintenant sur la tête d'un enfant ne se réalise pas avant un siècle.

Ces documents doivent être rédigés avee le plus grand soin, si l'on veut éviter les erreurs d'interprétation. Il est indispensable que rédacteurs et traducteurs sachent manier parfaitement leur langue et soient sensibles à toutes les exigences de la sémantique. Ces contrats ne constituent-ils pas pour l'assureur la principale garantie de rémunération de ses capitaux ? N'en est-il pas de même pour l'assuré, qui aurait à solder sa quote-part d'une erreur coûteuse par une diminution de ses participations?

Il est done grand temps que les dirigeants des compagnies d'assurance et les traducteurs s'entendent pour assurer le succès d'une politique de refrancisation qui s'impose.

Il faut avant tout que l'on cesse de s'autoriser des servitudes créées par les textes existants pour imposer aux traducteurs une syntaxe et des termes fautifs. On accrédite ainsi un moyen d'expression qui ne répond pas aux exigences de la technique des assurances et qui est de ce fait inapte au rôle qu'on veut lui faire tenir. Il faut aussi que les traducteurs, de leur côté, cherchent à apporter une solution définitive aux très nombreux problèmes d'ordre linguistique que pose le commerce des assurances sur la vie.

Comme contribution à l'effort commun, nous étudierons ci-après un terme fort usité, le mot benefit, qui est un peu le leitmotiv des textes d'assurance. Benefit signifie a payment or other assistance given by an insurance company. mutual benefit society, or public agency. Bien des choses en somme! 
Or, de son côté, bénéfice, dont on se sert en toutes occasions pour traduire l'expression en cause, signifie en français un gain réalisé dans une opération ou une entreprise (Cf. Robert). Lorsqu'on appelle bénéfice les gains réalisés et servis par les compagnies sous forme de participations, on parle français. Mais lorsqu'on dit « vous toucherez un bénéfice de $\$ 5000$ » au lieu de « un capital, une somme de $\$ 5000 »$, on peut se demander si on ne nous donnera pas de surcroît l'entreprise qui nous permettra de réaliser un tel gain !

Benefit se rend selon le cas par: avantage, prestation, garantie, somme, somme garantie, somme assurée, capital, assurance, rente, indemnité, profit, ete. Voici quelques indications sur l'emploi de ces expressions.

1 - Au sens général de «somme promise», on peut donner la priorité à prestation, qui a cours dans la législation sociale française depuis les années trente (Cf. Robert) et qui semble s'implanter depuis dans les assurances sur la vie. «Le montant de la prestation différée ainsi que le taux de l'intérêt à bonifier sur cette prestation sont fixés par la Compagnie» (Providentia de Genève). « Si la masse des primes est inférieure à la masse des prestations promises, il faut diminuer ces prestations 》 (Ancienne Mutuelle Vie de Rouen).

2-Lorsque benefit ne comporte pas uniquement l'idée de sommes assurées, garanties est tout indiqué. «Si l'assuré est alors vivant, il bénéficie des garanties ci-après » (L'Union de Paris). Dans le corps des avenants d'assurances complémentaires, e'est effectivement garanties que l'on donne comme titre à l'article où sont décrites les prestations et les modalités d'application. À noter également que garantie s'emploie aussi au singulier. «Si la police comporte la garantie du paiement du eapital assuré ...» (L'Union). "Avenant de garantie du risque d'invalidité »(Ibid.).

3 - Dans les contrats, il convient de rendre benefit par les termes qui le décrivent le mieux. Traduire, par exemple, the benefits provided under option $B$ par «les fractions mensuelles de capital et de rente prévues selon l'option $\mathrm{B} \gg$ apporte une précision utile pour l'intelligence du texte. En revanche, s'il est question de sommes immédiatement précitées, soit par exemple un capital assuré et des sommes résultant de différentes garanties complémentaires, on peut dire simplement les sommes, ou les sommes assurées, ou encore les sommes garanties. These benefits are payable only if the insured becomes totally disabled: Ces sommes ne deviennent exigibles que si l'assuré cst frappé d'invalidité totale.

4 - Benefit se retrouve dans de nombreuses expressions où il joue le rôle de déterminé. Mentionnons, accompagnées de leur traduction: Maturity benefit, Capital-échéance; Death benefit, Capital-décès; Family benefit, Assurance familiale.

5 - On entend par supplementary benefits des garanties qui viennent s'ajouter aux assurances prévues par le contrat principal. On les appelle assurances complémentaires ou encore garanties complémentaires. Elles font l'objet 
d'avenants dont on revêt la police. On peut donc leur donner le nom d'assurance, de garantie, d'avenant, d'additif, ou encore les désigner sans aucun déterminant.

Voici quelques-unes de ces garanties et la traduction que nous suggérons. Waiver of Premium Benefit, Avenant ou garantie d'exonération du paiement des primes. Accidental Death Benefit, Assurance en cas de décès par accident. Total Disability Benefit, Garantie du risque d'invalidité totale. Supplementary Term Insurance Benefit, Assurance temporaire supplémentaire. Family Income Benefit, Avenant de rente familiale ou simplement Rente familiale.

6 - Nous n'avons rien dit jusqu'à maintenant du mot indemnité. Greider et Beadles, dans Principles of Life Insurance, font la remarque que voici au sujet de cette expression: «This term, though widely used, is not technically correct, for life insurance is not a contract of indemnity ». En effet, l'assurance sur la vie n'est pas à caractère indemnitaire. Pour rendre en français Double Indemnity Benefit ou Triple Indemnity Benefit, disons simplement Assurance en cas de décès par accident ou encore Garantie double effet, ou triple effet, en cas de décès par accident. Toutefois, dans les autres assurances de personnes: invalidité, maladie, hospitalisation, etc., le mot indemnité est reçu.

7 -Insurance for the benefit of, Assurance au profit de. $\grave{A}$ noter qu'en franȩais on parle du bénéfice de l'assurance, non pour désigner les sommes assurées, mais la propriété, la jouissance du contrat. «Le consentement de l'assuré doit, à peine de nullité, être donné pour ... et pour le transfert du bénéfice $d u$ contrat souscrit sur sa tête par un tiers » (Loi du 12 juillet 1930, France). "Est exclue du bénéfice de la garantie toute personne qui, intentionnellement, aurait causé ou provoqué le sinistre (Ancienne Mutuelle Vie).

Que conclure des remarques qui précèdent? Que le français peut, si l'on s'en donne la peine, apporter à nos textes des précisions intéressantes, et qu'inversement une mauvaise traduetion peut comporter une perte d'information sérieuse. Le calque au niveau des mots et de la structure entrave le cheminement et la communication de la pensée; la présence d'un trop grand nombre de ealques dans un texte fait qu'on le comprend mal, ou qu'on ne le comprend pas du tout.

Une telle obseurité empêche l'assuré de bien comprendre les conditions de sa police et apporte de sérieux obstacles à la communication entre les employés de la Compagnie. Elle risque également de décourager ceux qui poursuivent des études en vue de faire carrière dans la vente des assurances sur la vie ou dans les bureaux de nos grandes sociétés.

On a done raison de souligner les faiblesses de la langue des assurances en milieu canadien-français, et il convient de se demander sérieusement si des chances de redressement existent.

Disons qu'il y a lieu d'espérer. Des efforts individuels ont déjà été tentếs. Un certain nombre de compagnies anglophones ont accepté une bonne partie de ce que nous pourrions appeler la nouvelle terminologie. Chapeau bas aux dirigeants de ces sociétés, car ils ont fait preuve d'un sens pratique qui les 
honore. D'autre part, les grands organismes canadiens qui, sur le plan des assurances sur la vie, correspondent plus ou moins à ce qu'en France on appelle l'École nationale d'Assurances, témoignent d'une bonne volonté manifeste et ils emboîteront volontiers le pas, croyons-nous, à tout mouvement de refrancisation qui aura reçu caution en haut lieu.

La façon d'amorcer ce mouvement? Établir d'abord un glossaire anglais-français qui tiendra rigoureusement compte des exigences du français universel et des particularités de la pratique des assurances sur la vie au Canạda et aux États-Unis.

Cet ouvrage devra fournir un répertoire aussi complet que possible des nombreux termes et expressions ayant cours dans le domaine des assurances.

Il devra être explicite; offrir en abondance des définitions, des traductions de phrases types, des citations tirées des meilleurs traités rédigés dans les pays francophones; s'adresser aussi bien aux simples employés de bureau et aux cadres qu'aux traducteurs et aux rédacteurs. Il devra être pratique et de consultation facile. Les différentes rubriques devraient être classées selon l'ordre alphabétique des mots anglais; un index des mots français permettrait d'effectuer des recherches et vérifications en sens inverse. Une bibliographie terminerait ce volume.

Il conviendrait également d'y faire figurer, à l'occasion, des notions de stylistique comparée, seule façon de mettre en lumière la démarche propre au français et de provoquer le souci de la sobre élégance qui doit caractériser les textes des grandes sociétés commerciales.

Par la suite, il serait sans doute assez facile de traduire un traité qui servirait de base aux manuels des différents cycles d'étude.

C'est à ce prix, croyons-nous, et à ce prix seulement, que nous pourrons refranciser la pratique des assurances sur la vie au Canada.

En attendant qu'un tel ouvrage existe, le traducteur doit travailler de son côté à améliorer la situation. Il le fera en renonçant à la facilité, en se documentant soigneusement, et en persistant dans son désir de mieux dire.

Jean-Paul de Grandrré Montréal 